\title{
Non-destructive 3D geometric modeling of maize root-stubble in-situ via X-ray computed tomography
}

\author{
Xu Zhao*, Luyu Xing, Shifan Shen, Jiaming Liu, Daixing Zhang \\ (School of Mechanical Engineering \& Automation, University of Science and Technology Liaoning, Anshan 114051, China)
}

\begin{abstract}
No-tillage seeding has become an important approach to improve crop productivity, which needs colters of high performance to cut the root-stubble-soil composite. However, the difficulty of maize root-stubbles three-dimensional (3D) modeling hinders finite element (FE) simulation to improve development efficiency of such colters because of maize root system complexity and opaque nature of the soil. Fortunately, the non-destructive 3D geometric model of the maize root-stubble in-situ can be established via X-ray computed tomography (CT) following by a systematic procedure. The whole procedure includes CT scanning of the maize root-stubble-soil composite sample, image reconstruction via filtered back-projection (FBP) with the Hanning filter, segmentation of root-stubble via a variational level set method, and post-processing via morphological operations. The 3D reconstruction model of the maize root-stubble in-situ presents a complete, complex and in-situ geometrical morphology, which cannot be realized via other methods, including the destructive modelling after washing via CT. This study is the first to build a 3D geometric model of a maize root-stubble in-situ via CT, which opens up new possibilities for simulation of root-stubble-soil cutting using FEM, and much other research related to plant root-stubbles.
\end{abstract}

Keywords: maize root-stubble, non-destructive modeling, X-ray computed tomography, variational level set method DOI: $10.25165 /$ j.ijabe.20201303.5268

Citation: Zhao X, Xing L Y, Shen S F, Liu J M, Zhang D X. Non-destructive 3D geometric modeling of maize root-stubble in-situ via X-ray computed tomography. Int J Agric \& Biol Eng, 2020; 13(3): 174-179.

\section{Introduction}

No-tillage seeding plays a crucial role in the increase of crop production via leaving residue cover to protect the field, but which causes plugging of seeders. No-tillage seeder has been developed to overcome the dilemma, whose core components are mostly colters which cut soil and root-stubble ${ }^{[1]}$. The development of high performance colters needs FEM simulation to analyze dynamic cutting problems of colters on the root-stubble-soil composite regarding material failure ${ }^{[2]}$. However, such FE simulations cannot be conducted effectively so far because the accurate 3D geometric model of maize root-stubble is unable to be established due to root system complexity and opaque nature of the soil. The most common method used to establish the 3D geometric model of a root-stubble is invasive and destructive, such as root-stubble shovelomics, including root-stubble washing, manual measurement and extremely simplified modeling, such as the literate ${ }^{[3]}$. However, such methods all result in root breakage, position and pose change during the washing process, as well as severe inexactness during simplified modeling, due to complexity and fragile of dried roots, such inaccurate geometric modeling of

Received data: 2019-07-08 Accepted data: 2020-03-20

Biographies: Luyu Xing, Postgraduate, research interests: no-till planting techniques and image processing, Email: 2056846657@qq.com; Shifan Shen, Postgraduate, research interests: bionic engineering, Email: 605941959@qq.com. Jiaming Liu, Postgraduate, research interests: no-till planting techniques, Email: 935373952@qq.com; Daixing Zhang, Master, Lectturer, research interests: no-till planting techniques, zdxing2005@126.com.

*Corresponding author: Xu Zhao, Master, Associate Professor, research interests: no-till planting techniques and numerical modeling of soil-plant-machine system. School of Mechanical Engineering \& Automation, University of Science and Technology Liaoning, Anshan 114051, China. Email: 1982zhaoxu@163.com. the root-stubble make the validity of such FE cutting simulation to be problematic.

Fortunately, X-ray computed tomography (CT) can realize the nondestructive visualization of root systems within the surrounding soil ${ }^{[4]}$. Mooney et al. ${ }^{[5]}$ applied CT to visualize and quantify root lodging of wheat within different kinds of soil. Kaestner et al. ${ }^{[6]}$ presented a method which including three steps to realize the reconstruction of the root network. Lontoc-Roy et al ${ }^{[7]}$ used CT to scan maize root systems in different soil surroundings, and an algorithm was developed in MATLAB based on CT histograms to segment root from surrounding soil, ultimately, they reconstructed the maize root systems. Perret et al. ${ }^{[8]}$ visualized and quantified roots within soil via $\mathrm{CT}$ based on optimization of root-soil threshold. Han et al. ${ }^{[9]}$ utilized CT to scan the potato below ground and extracted roots via histogram analysis of CT numbers. Hargreaves et al. ${ }^{[10]}$ analyzed 2D characteristics of barley seedling roots within gel chambers via CT, the study did resolve the root isolation form soil. Mairhoffer et al. ${ }^{[11]}$ used RooTrak to realize the root-soil segmentation, and in another study Mairhoffer et al. ${ }^{[4]}$ conducted a comparison of root-soil segmentation tools, the conclusion was that VGStudio Max and RooTrak were qualified for the mission. Koebernick et al. ${ }^{[12]}$ used a region growing algorithm to realize the root-soil segmentation from $\mathrm{CT}$, and a single Vicia faba L. root systems in a sandy soil was visualized nondestructively. Very recently, $\mathrm{Xu}$ et al. ${ }^{[13]}$ reviewed statistical and computational approaches that had been or might be effective for the analysis of 3D CT images of plant roots, on the aspects of root segmentation, root-system reconstruction and extraction of higher-level phenotypes. Tabb et al. ${ }^{[14]}$ presented a method based on level sets to achieve the isolation of plant roots from the soil. Maenhout et al. ${ }^{[15]}$ successfully extracted mature maize roots from CT volumes of real root-soil composites via a semi-automated segmentation method operated in VGStudio Max 3.2. Zheng et 
al. ${ }^{[16]}$ provided a review of approaches on analysis of plant rootsbased CT, including the root isolation from the soil, root architecture reconstruction.

In summary, a number of studies were conducted to visualize plant root systems within soil via CT, such progress offers significant benefits for non-destructive 3D geometric modeling of maize root-stubble in-Situ via CT. However, previous studies were carried out for living plant roots within the soil, additionally, visualized plant roots were mostly buried or grown in artificially prepared sand, which was different from the field soil. Little research has been done on CT scanning of dried maize root-stubble in-situ after harvest, in comparison to living plant roots, more similar densities of dried maize roots and soil cause overlap of CT grey values between roots and soil, which hinders the root-soil segmentation. The study focuses on image reconstruction, root-soil segmentation and post-processing during 3D modeling of dried maize root-stubble in-situ via CT.

\section{Materials and Methods}

\subsection{Sample preparation}

The maize root-stubble-soil composite sample (see Figure 1), whose volume of the earthwork cuts was $350 \mathrm{~mm}$ in height $220 \mathrm{~mm}$ in diameter, were dug undisturbedly and randomly from harvested maize field in April 2019. After dug, the sample was immediately sealed in a vacuum-sealed bag until CT scanning.

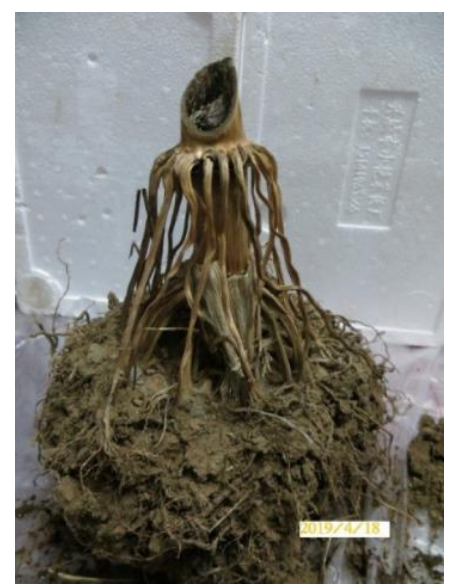

Figure 1 Maize root-soil-stubble composite sample

2.2 X-ray computed tomography scanning configuration and measurements

X-ray CT scanning is conducted on the CT scanner (Neviz-64e) with 64-row detectors using a voltage of $80-140 \mathrm{~kW}$ and a current of automatic 20 500 Ma, as shown in Figure 2. $460 \mathrm{CT}$ slices of $0.1 \mathrm{~mm}$ thick cross-section is produced via $\mathrm{X}$-ray CT scanning.

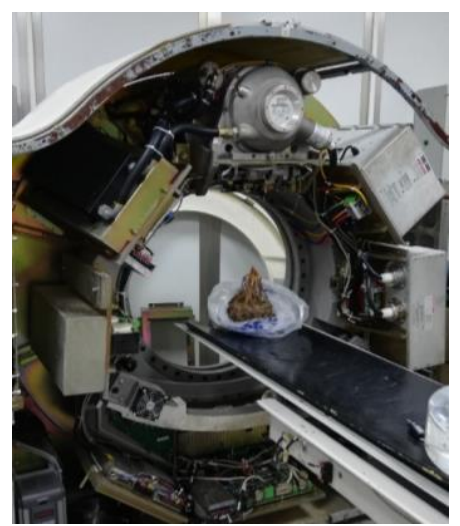

Figure 2 Maize root-soil-stubble composite sample on the couch of the CT scanner
In the study, the calibrated CT number (CTN) value of air is set as $-1000 \mathrm{HU}, \mathrm{CTN}^{[17]}$ is described as

$$
\mathrm{CTN}(\mathrm{HU})=1000\left(\mu-\mu_{w}\right) /\left(\mu_{w}-\mu_{a}\right)
$$

where, $\mu_{w}$ and $\mu_{a}$ are the attenuation coefficient of X-rays through the water and air, respectively.

In our operations, CT parameters are set as follow: $140 \mathrm{~kW}$ for the voltage and automatic $20-500 \mathrm{~mA}$ for the current.

\subsection{Image reconstruction}

In this study, 3D filtered back-projection (FBP) reconstruction algorithm ${ }^{[18]}$ is used to conduct image reconstruction, including data filtering and back-projection. Back-projection of CT data causes diffusion of the CT image, which is corrected via a Hanning filter, as follows:

$$
H(\omega)=\left\{\begin{array}{cc}
0.5+0.5 \cos \left(\frac{\pi \omega}{\omega_{m}}\right), & 0 \leq|\omega| \leq \omega_{m} \\
0, & \text { otherwise }
\end{array}\right.
$$

where, $\omega$ and $\omega_{m}$ denote the spatial frequency and the cut-off frequency, respectively.

As shown in Figure 3, the object is scanned in the coordinate system $(x, y, z), \Phi, F$ and $s$ denote the detection angle, focal length and the distance between the focal point and the origin, respectively.

According to Literature [18], CTN can be reconstructed as follows:

$$
\begin{aligned}
& f(x, y, z)= \\
& \int d \Phi \int_{-R}^{R} d Y^{\prime} p_{\Phi}\left(Y^{\prime}, Z\right) \frac{s}{\left(F^{2}+Y^{\prime 2}\right)^{1 / 2}} \frac{F^{2}}{(s+x \cos \Phi+y \sin \Phi)^{2}} H\left(Y-Y^{\prime}\right)
\end{aligned}
$$

where

$$
Y=\frac{F(-x \sin \Phi+y \cos \Phi)}{s+x \cos \Phi+y \sin \Phi}
$$

and $Z=z, p_{\Phi}\left(Y^{\prime}, Z\right)$ is the value of the projections at $\left(Y^{\prime}, Z\right)$ on the detector surface and $R$ is the value of $Y^{\prime}$ for which $p_{\Phi}\left(Y^{\prime}, Z\right)=0$ with $\left|Y^{\prime}\right|>R . \quad H(t)$ is the Hanning filter.

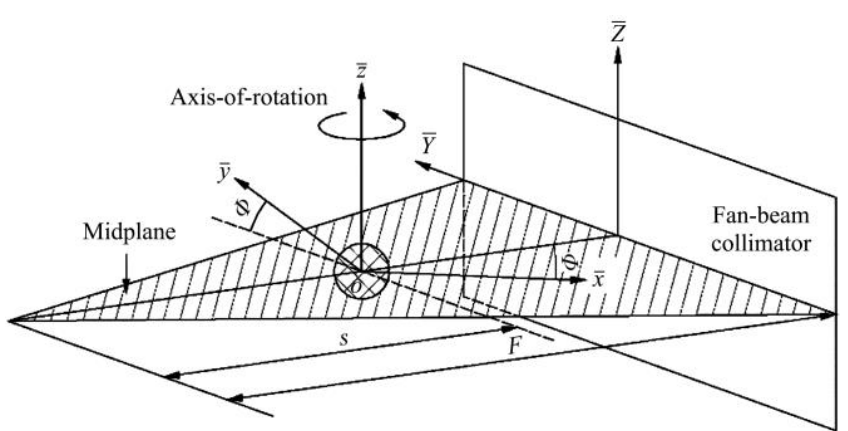

Figure 3 Three-dimensional coordinate system for a fan-beam geometry

\subsection{Root-stubble segmentation}

The segmentation of maize root-stubble from soil and matters in $\mathrm{CT}$ images is a challenging problem because a dried maize root-stubble shares similar density with surrounding non-root matters, and density of maize root-stubble regions is inhomogeneity, thus the frequently-used thresholding segmentation method cannot effectively achieve the segmentation. To overcome the problem, the local binary fitting energy (LBF) model in a variational level set method, proposed by $\mathrm{Li}$ et al. ${ }^{[19]}$, is adopted for the segmentation of maize root-stubble.

Assume the image domain $\Omega$ is divided into two regions: $\Omega_{1}=$ outside $(C)$ and $\Omega_{2}=$ inside $(C)$, where $C$ is a closed contour 
represented by a level set Lipschitz function $\phi: \Omega \rightarrow \mathfrak{R}$, then the energy function $\varepsilon^{L B F}\left(\phi, f_{1}(x), f_{2}(x)\right)$ can be expressed as

$$
\begin{aligned}
\varepsilon^{L B F} & =\left(\phi, f_{1}(x), f_{2}(x)\right) \\
& =\sum_{i=1}^{2} \lambda_{i} \int\left(\int K_{\sigma}(x-y)\left|I(y)-f_{i}(x)\right|^{2} M_{i}^{\epsilon}(\phi(y)) d y\right) d x
\end{aligned}
$$

where, $\lambda_{1}$ and $\lambda_{2}$ are positive constants; $f_{1}(x)$ and $f_{2}(x)$ represent the proximate image at point $x$ in $\Omega_{1}$ and $\Omega_{2}$, respectively; $I(y)$ are the effective intensities; $K_{\sigma}$ is a kernel function with $\sigma>0$ expressed as follows:

$$
K_{\sigma}(s)=\frac{1}{\sqrt{2 \pi} \sigma} e^{-|s|^{2}} / 2 \sigma^{2}
$$

and $M_{1}^{\epsilon}(\phi)=H_{\epsilon}(\phi), M_{2}^{\epsilon}(\phi)=1-H_{\epsilon}(\phi), H_{\epsilon}$ is a smooth function for approximating the Heaviside function $H$, expressed by

$$
H_{\epsilon}(x)=\frac{1}{2}\left[1+\frac{2}{\pi} \arctan \left(\frac{x}{\epsilon}\right)\right]
$$

its derivative is

$$
\delta_{\epsilon}(x)=H_{\epsilon}^{\prime}(x)=\frac{1}{\pi} \frac{\epsilon}{\epsilon^{2}+x^{2}}
$$

Then the variational level set can be expressed as

$$
\mathcal{F}\left(\phi, f_{1}(x), f_{2}(x)\right)=\varepsilon^{L B F}\left(\phi, f_{1}(x), f_{2}(x)\right)+v \mathcal{L}(\phi)+\mu \mathcal{R}(\phi)
$$

where, $v$ and $\mu$ are positive constants; $\mathcal{L}(\phi)$ and $\mathcal{R}(\phi)$ are regularization terms defined by

$$
\begin{gathered}
\mathcal{L}(\phi)=\int\left|\nabla H_{\epsilon}(\phi(x))\right| d x \\
\mathcal{R}(\phi)=\int \frac{1}{2}(|\nabla \phi(x)|-1)^{2} d x
\end{gathered}
$$

The image segmentation is realized via minimizing $\mathcal{F}\left(\phi, f_{1}(x), f_{2}(x)\right)$. For fixed $\phi, \quad \mathcal{F}\left(\phi, f_{1}(x), f_{2}(x)\right)$ is minimized via optimal $f_{1}(x)$ and $f_{2}(x)$ expressed as follows:

$$
f_{i}=\frac{K_{\sigma}(x) *\left[M_{i}^{\epsilon}(\phi(x)) I(x)\right]}{K_{\sigma}(x) * M_{i}^{\epsilon}(\phi(x))} \quad i=1,2
$$

where, $f_{i}(x)$ is the weighted mean intensity in a neighborhood with the center $x$ and radius $\sigma$. And for fixed $f_{1}(x)$ and $f_{2}(x)$, $\mathcal{F}\left(\phi, f_{1}(x), f_{2}(x)\right)$ is minimized by solving Equation (13).

$$
\begin{aligned}
& \frac{\phi^{k+1}-\phi^{k}}{\Delta t}=\frac{\partial \phi}{\partial t}=-\sigma_{\epsilon}\left(\phi^{k}\right)\left(\lambda_{1} e_{1}-\lambda_{2} e_{2}\right)+ \\
& v \delta_{\epsilon}\left(\phi^{k}\right) \operatorname{div}\left(\frac{\nabla \phi^{k}}{\left|\nabla \phi^{k}\right|}\right)+\mu\left(\nabla^{2} \phi^{k}-\operatorname{div}\left(\frac{\nabla \phi^{k}}{\left|\nabla \phi^{k}\right|}\right)\right)
\end{aligned}
$$

where, $\sigma_{\epsilon}$ is given by Equation (8); $e_{1}$ and $e_{2}$ are described as

$$
e_{i}(x)=\int K_{\delta}(y-x)\left|I(x)-f_{i}(y)\right|^{2} d y
$$

where, $f_{i}(y)$ is given by Equation (12).

The numerical algorithm can be written as the following 4 steps:

1) Set $k=1$ and the level set function $\phi$ is initialized as follows:

$$
\phi(x, 0)= \begin{cases}-c_{0}, & x \in R_{0} \\ c_{0}, & x \notin R_{0}\end{cases}
$$

where, $c_{0}$ is a positive constant, which is set as $c_{0}=1$ in the study.

In the initial stage of the segmentation, a root-stubble region in the CT image should be selected arbitrarily, marked as $R_{0}$.

2) Compute $f_{i}(x)$ from Equation (12).

3) Solve $\phi$ Equation (13).

4) Estimate whether the calculation converges, if so, update the maize root-stubble histogram, if not, set $k=k+1$ and return to step 2 .

\subsection{Post-processing}

Due to the existence of defects after the image segmentation, such as disconnections, isolated fragments or small holes, morphological operations are adopted to conduct post-processing. Because maize root-stubble and non-root matters fall into some overlap of gray-level intensities, the segmented root-stubble images contain non-root matters, thus erosion operation should be used to remove such non-root matters, closing operations can fill out the interior of the root-stubble images. The above morphological operations are described in Literature [20].

\subsection{Destructive analysis of the maize root-stubble}

As shown in Figure 4, the maize root-stubble was extracted from the above maize root-stubble-soil composite sample which had been scanned via CT by washing and analyzed to verify the non-destructive 3D geometric modeling of maize root-stubble in-situ via CT. The washed maize root-stubble was also scanned via CT to establish its destructive geometric model, quantify specific parameters (i.e., total volume, total length, the maximum width, the minimum root radium, etc.), such destructive geometric model and measurements are then compared with the output of the non-destructive 3D geometric modeling in-situ via CT.

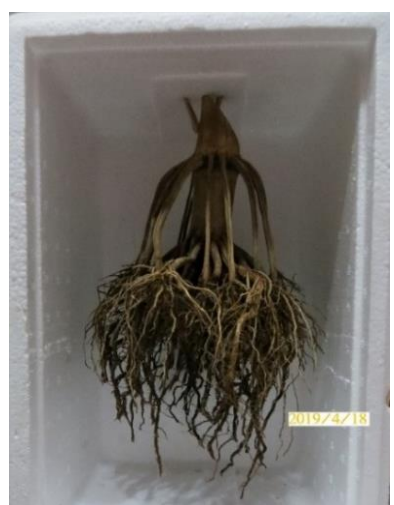

Figure 4 Washed maize root-stubble extracted from the above sample

\section{Results and discussion}

\subsection{Implementation of FBP for image reconstruction of the maize root-stubble-soil composite}

460 CT images of the maize root-stubble-soil composite are reconstructed via FBP, where the cut-off frequency of the Hanning filer is set equal to the Nyquist frequency $(2.0$ cycles $/ \mathrm{cm})$, namely half of the sampling rate.

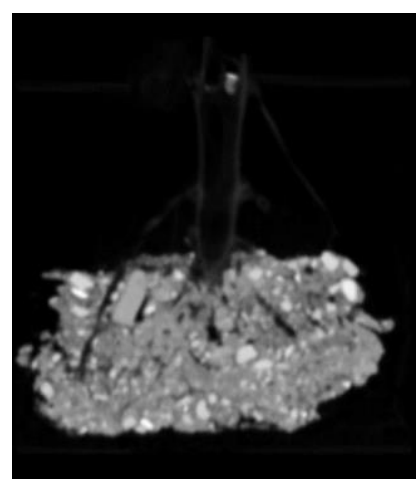

a. Without filtering

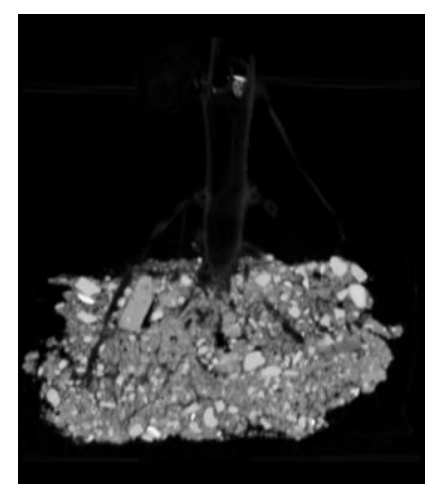

b. With filtering
Figure 5 Transverse CT slice 205 of the maize root-soil-stubble composite

Take the CT slice 205 of the maize root-stubble-soil composite as an example, the image without filtering shown in Figure $5 \mathrm{a}$ is more diffuse than the image with the above filtering shown in 
Figure 5b. The comparison demonstrates that pre-filtering is necessary for CT image reconstruction. As expected, FBP with the Hanning filter, whose cut-off frequency is 2.0 cycles/cm, proves to be effective for image reconstruction of the maize root-soil-stubble composite.

\subsection{Implementation of the variational level set method for root-stubble segmentation}

Based on the experience of Literature [19], the parameters of the LBF model in a variational level set method are set as follows: $\sigma=3.0, \epsilon=1, \lambda_{1}=\lambda_{2}=1, v=0.001 \times 255^{2}, \mu=1, \Delta t=0.1, \quad$ and the termination occurs when $\frac{\left|\phi^{k+1}-\phi^{k}\right|}{\left|\phi^{k}\right|} \leq 0.001$ and $\left|\phi^{k+1}\right| \leq 0.001$, Li et al. ${ }^{[19]}$ stated that a smaller scale $\sigma$ can produce a more accurate location of the object boundaries. In order to present $\Omega_{1}$ and $\Omega_{2}$ voxels from being added to either, the minimum grayscale intensity value in $\Omega_{1}$ and $\Omega_{2}$ are set as -900 and -350 based on pre-analysis of the approximate grayscale intensity value of maize root-stubble from CT images. The above implementations contribute to overcoming overlap between root-stubble and non-root matters.

Figure 6 shows the segmentation results of root-stubbles from the soil and other matters for CT slice 200 (Figure 6d), CT slice 240 (Figure 6e) and CT slice 320 (Figure 6f). These results demonstrate that the segmentation method performs well on the capture of the desirable root-stubble vessels, including small root hair and the parts of degraded intensity values. Still, the method has difficulties in segmenting the interacting roots.

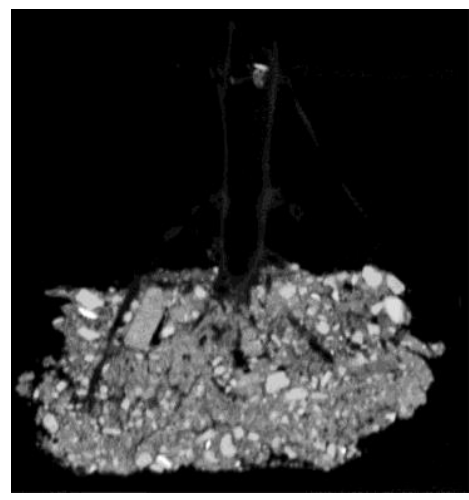

a. Original CT slice 200

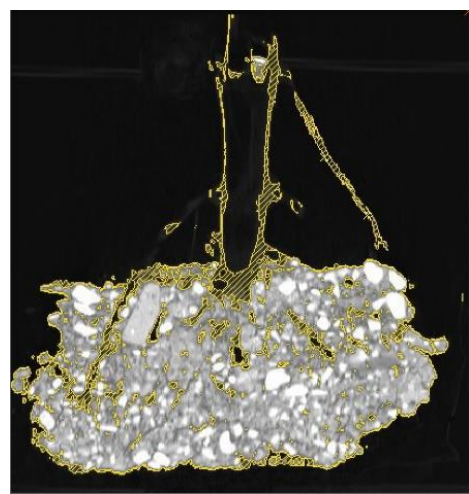

d. Final segmented CT slice 200

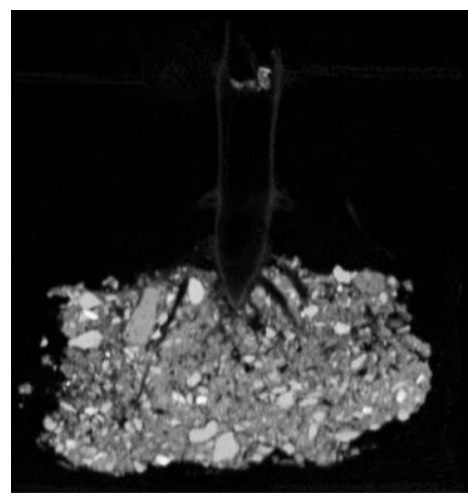

b. Original CT slice 240

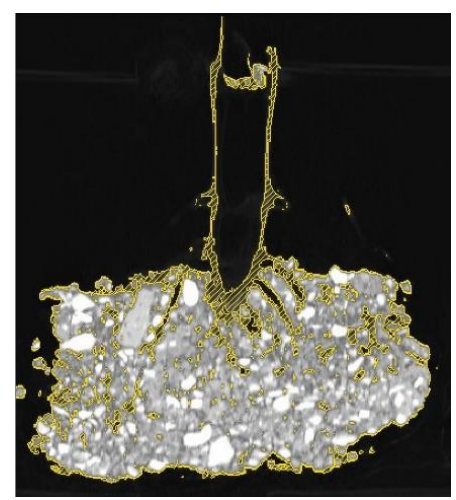

e. Final segmented CT slice 240

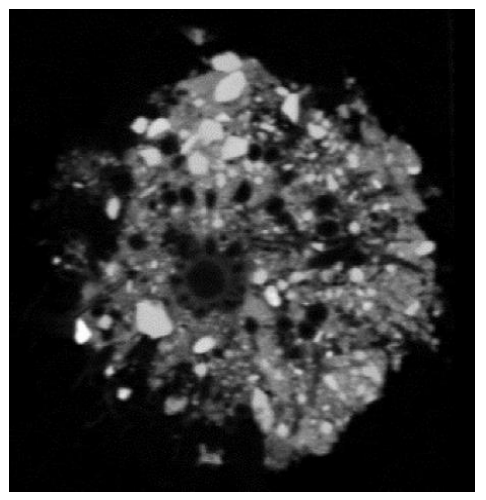

c. Original CT slice 320

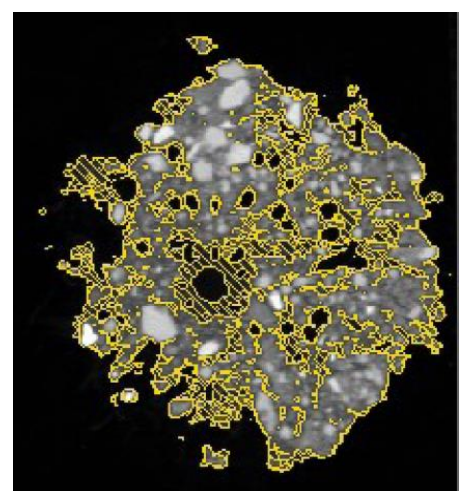

f. Final segmented CT slice 320

Figure 6 Segmentation results of root-stubble from soil and other matters for CT slices

\subsection{Implementation of morphological operations}

Figure 7 shows a case study of morphological operations on one detail of the non-destructive 3D geometric model of the maize root-stubble via CT. Because of overlap of gray-level intensities between root-stubble and non-root matters and intensities degradation, some defects, including partial missing, discontinuity,

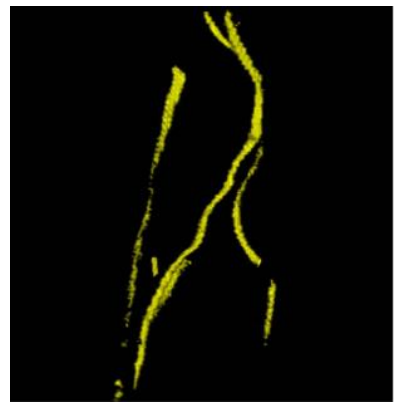

a. Without morphological operations

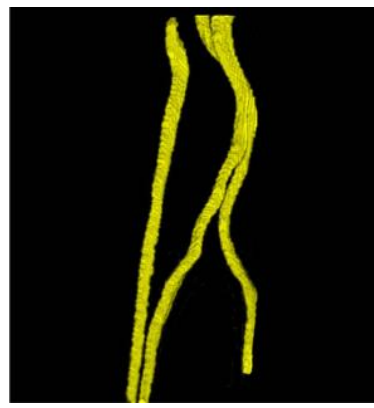

b. With morphological operations
Figure 7 One detail of the non-destructive 3D geometric model of the maize root-stubble via CT and the existence of non-root matters, appear after the segmentation, as shown in Figure 7a. Then a morphological closing operation is used to correct the parts missing and discontinuity of the root-stubble, a morphological erosion operation is utilized to eliminate the non-root matters, consequently, the resultant effect is shown as Figure $7 \mathrm{~b}$.

3.4 Non-destructive $3 \mathrm{D}$ geometric modeling of the maize root-stubble in-situ via $\mathrm{CT}$

Figure 8 shows the 3D reconstructed model of the maize root-stubble in-situ segmented from the soil and other growing media via CT by the following procedures: CT scanning of the maize root-stubble-soil composite, image reconstruction via FBP with the Hanning filter, segmentation of root-stubble via the above variational level set method, and post-processing via morphological operations. The model can be interactively operated, such as rotation and cut. Figure $8 \mathrm{a}$ shows the complex complete architecture of maize root-stubble with numerous lateral roots which play a crucial role in the root-stubble architecture.

After harvest, the structure of dried maize root-stubble is 
different from that of well-hydrated maize root and stalk. The stalk of dried root-stubble presents composite structure including the outer fibrous epidermis and inner flocculent pith, as shown in Figures $8 \mathrm{a}$ and $\mathrm{b}$. Hollow structure is the most common form of

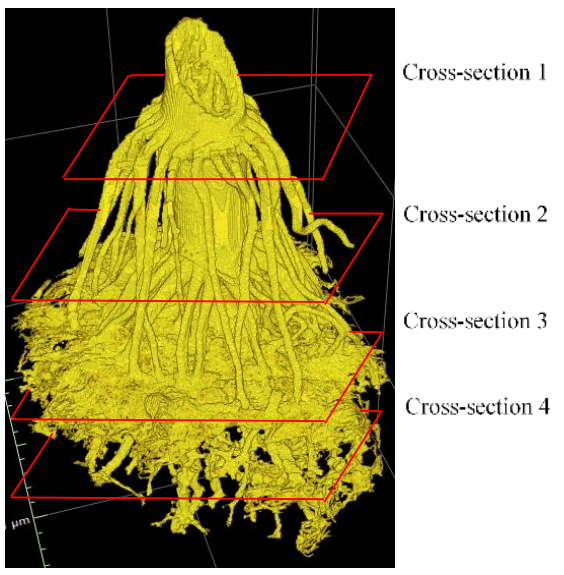

a. Complete maize root-stubble

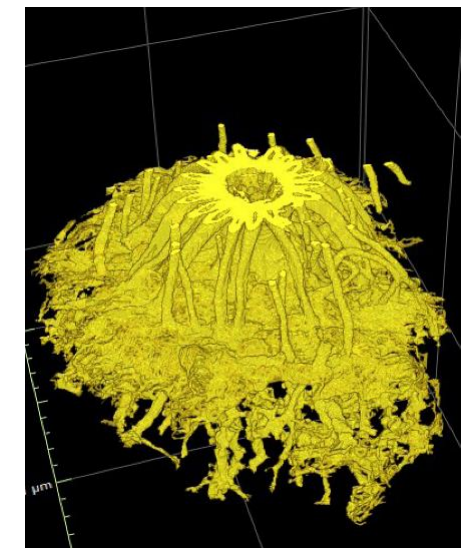

c. Cross-section 2 which is marked in Figure 8a

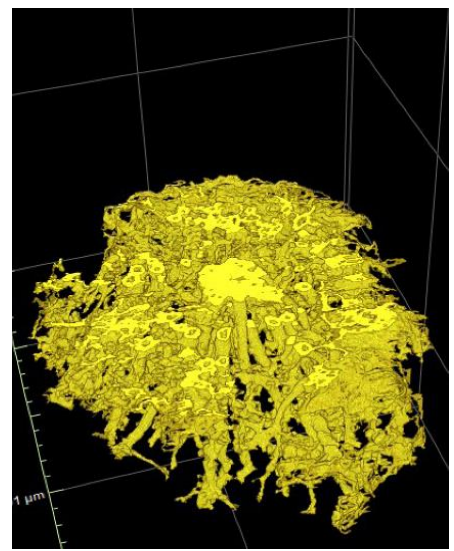

d. Cross-section 3 which is marked in Figure 8a the dried maize root, such as brace roots are shown in Figure 8a, crown roots are shown in Figure $8 \mathrm{~b}$, primary root is shown in Figure $8 \mathrm{c}$, and seminal roots and lateral roots are shown in Figure $8 d$.

Figure 8 3D reconstruction model of the maize root-stubble segmented from soil and other growing media via CT

3.5 Destructive 3D geometric modeling of the maize root-stubble after washing via $\mathrm{CT}$

After washing, the maize root-stubble extracted from the above maize root-stubble-soil composite sample is scanned via CT, then the destructive 3D model is obtained as shown in Figure 9.

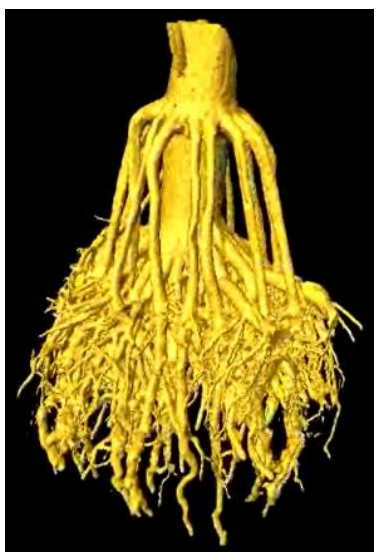

Figure 9 Destructive 3D model of the maize root-stubble after washing via $\mathrm{CT}$

Compared to the non-destructive 3D model of the maize root-stubble in-situ shown in Figure 8, the destructive 3D model owns two defects. One is the incomplete architecture, including the loss of abundant tiny roots and a number of thick roots, as well as roots breakages which result from the inherent fragility of dried roots and the inevitable destruction effect of washing. The other one is the change of roots position and pose, namely, all roots droop due to gravity and none of the support provided by the field soil.

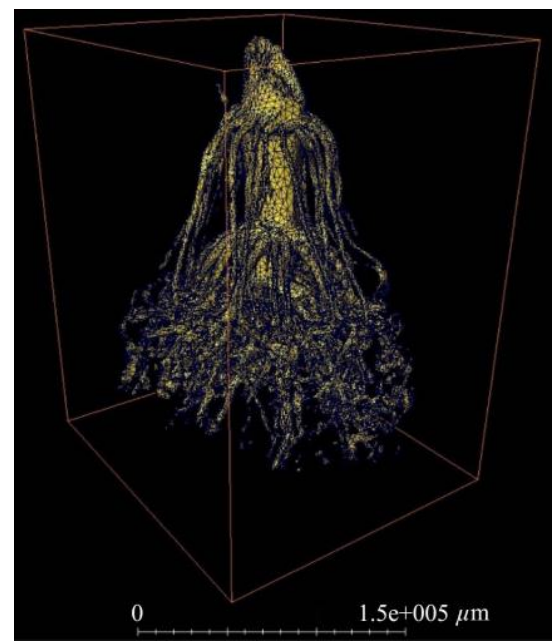

Figure 10 STL format of the 3D non-destructive model of the maize root-stubble in-situ via $\mathrm{CT}$

Furthermore, Table 1 compares the specific parameters of the non-destructive model in-situ and the destructive model after washing. It can be observed that the non-destructive model in-situ records a significant increment of $28.03 \%$ in total volume, 
$1012.15 \%$ in total length (summation of all maize root length) and $24.24 \%$ maximum width, but significant decrement of $27.89 \%$ in minimum root radium, compared to the destructive model after washing.

Table 1 Quantitative evaluation of maize root-stubble characteristics via CT scanning in-situ and after washing

\begin{tabular}{lcccc}
\hline & $\begin{array}{c}\text { Total } \\
\text { volume } / \mu \mathrm{m}^{3}\end{array}$ & $\begin{array}{c}\text { Total } \\
\text { length } / \mu \mathrm{m}\end{array}$ & $\begin{array}{c}\text { Maximum } \\
\text { width } / \mu \mathrm{m}\end{array}$ & $\begin{array}{c}\text { Minimum root } \\
\text { radium } / \mu \mathrm{m}\end{array}$ \\
\hline $\begin{array}{l}\text { CT scanning in-situ } \\
\text { CT scanning after }\end{array}$ & $5.07 \mathrm{E}+014$ & $1.19 \mathrm{E}+007$ & $2.05 \mathrm{E}+005$ & 808.4 \\
washing & $3.96 \mathrm{E}+014$ & $1.07 \mathrm{E}+006$ & $1.65 \mathrm{E}+005$ & 1551.4 \\
\hline
\end{tabular}

The above comparison analyses indicate that the non-destructive model in-situ via CT preserves the natural characteristics of the maize root-stubble, such as complete structure, position and pose in situ within the field soil environment, which is achieved via invasive or destructive methods, such as shovelomics.

Finally, the 3D non-destructive model of the maize root-stubble in-situ via $\mathrm{CT}$ can be transformed into stereolithography (STL) format, as shown in Figure 10, which is supported by FE software packages, so 3D dynamic FEM simulation of root-stubble-soil cutting will be more accurately conducted.

\section{Conclusions}

3D geometric modeling of the maize root-stubble is essential for the root-stubble-soil composites cutting simulation during no-tillage seeding machinery's colters analyses. In the study, the non-destructive 3D geometric model of the maize root-stubble in-situ is established via CT by the following procedures: CT scan of the maize root-stubble-soil composite, image reconstruction via FBP with the Hanning filter, segmentation of root-stubble via the level set method, and post-processing via morphological operations. The non-destructive maize root-stubble model in-situ is ultimately transformed into STL format which can be supported by FE software packages.

Comparisons of images and structural parameters between the non-destructive model in-situ via CT and the destructive model after washing via $\mathrm{CT}$ show that the $\mathrm{CT}$ scan of the maize root-stubble-soil composite following by the data processing techniques used in the study produces more a complete and accurate 3D geometric model of the maize root-stubble in-situ.

However, the used method in the study is not very effective in extracting multiple interacting lateral roots due to poor precision of $\mathrm{CT}$ and the segmentation algorithm, in the future study, we will focus on the extracting algorithm of multiple interacting roots and high-resolution CT application.

\section{Acknowledgements}

The work is financially supported by the National Natural Science Foundation of China (Grant No. 51705227), University of Science and Technology Liaoning Talent Project Grants (Grant No. 601011507-19) and Special Scientific Research Foundation of University of Science and Technology Liaoning (Grant No. 2016YY02). We thank Jiuming Su for his lab assistance, and also thank Zong Zhang and Xinrui Xu for their analysis assistance.

\section{[References]}

[1] Zeng, Z W, Chen Y. The performance of a fluted coulter for vertical tillage as affected by working speed. Soil and Tillage Research, 2018; 175: 112-118.

[2] Upadhyaya S K., Rosa U A., Wulfsohn D. Application of the finite element method in agricultural soil mechanics. Advances in Soil Dynamics, 2002; 2: 117-153.

[3] Li M, Xu S, Yang Y W, Guo L, Tong J. A 3D simulation model of corn stubble cutting using finite element method. Soil and Tillage Research, 2017; 166: 43-51.

[4] Mairhofer S, Sturrock C, Wells D M., Bennett M J, Mooney S J, Pridmore T P. On the evaluation of methods for the recovery of plant root systems from X-ray computed tomography images. Functional Plant Biology, 2015; 42(5): 460.

[5] Mooney S J, Morris C, Berry P M. Visualization and quantification of the effects of cereal root lodging on three-dimensional soil macrostructure using X-ray computed tomography. Soil science, 2006; 171(9): 706-718.

[6] Kaestner A, Schneebeli M, Graf F. Visualizing three-dimensional root networks using computed tomography. Geoderma, 2006; 136(1-2): 459-469.

[7] Lontoc-Roy M, Dutilleul P, Prasher S O, Han L, Brouillet T, Smith D L. Advances in the acquisition and analysis of CT scan data to isolate a crop root system from the soil medium and quantify root system complexity in 3-D space. Geoderma, 2006; 137(1-2): 231-241.

[8] Perret J S, Al-Belushi M E, Deadman M. Non-destructive visualization and quantification of roots using computed tomography. Soil Biology and Biochemistry, 2007; 39(2): 391-399.

[9] Han L, Dutilleul P, Prasher S O, Beaulieu C, Smith D L. Assessment of density effects of the common scab-inducing pathogen on the seed and peripheral organs of potato during growth using computed tomography scanning data. Transactions of the ASABE, 2009; 52(1): 305-311.

[10] Hargreaves C E, Gregory P J, Bengough A G. Measuring root traits in barley (Hordeum vulgare ssp. vulgare and ssp. spontaneum) seedlings using gel chambers, soil sacs and X-ray microtomography. Plant and Soil, 2009; 316(1-2): 285-297.

[11] Mairhofer S, Zappala S, Tracy S, Sturrock C, Bennett M. J, Mooney S. J, et al. Recovering complete plant root system architectures from soil via X-ray $\mu$-Computed Tomography. Plant Methods, 2013; 9(1): 8.

[12] Koebernick N, Weller U, Huber K, Schlüter S, Vogel H J, Jahn R, et al. In situ visualization and quantification of three-dimensional root system architecture and growth using X-ray computed tomography. Vadose Zone Journal, 2014; 13(8). doi: 10.2136/vzj2014.03.0024

[13] $\mathrm{Xu} \mathrm{Z,} \mathrm{Valdes} \mathrm{C,} \mathrm{Clarke} \mathrm{J.} \mathrm{Existing} \mathrm{and} \mathrm{potential} \mathrm{statistical} \mathrm{and}$ computational approaches for the analysis of 3D CT images of plant roots. Agronomy, 2018; 8(5): 71.

[14] Tabb A, Duncan K E, Topp C N. Segmenting root systems in X-ray computed tomography images using level sets. In: 2018 IEEE Winter Conference on Applications of Computer Vision (WACV), Lake Tahoe: IEEE, 2018; pp.586-595.

[15] Maenhout P, Sleutel S, Xu H, Van Hoorebeke L, Cnudde V, De Neve S. Semi-automated segmentation and visualization of complex undisturbed root systems with X-ray $\mu \mathrm{CT}$. Soil and Tillage Research, 2019; 192: 59-65.

[16] Zheng X, Valdes C, Clarke J. Existing and potential statistical and computational approaches for the analysis of 3D CT images of plant roots. Agronomy, 2018; 8(5): 71.

[17] Kalender W A. Computed tomography: Fundamentals, system technology, image quality, applications. John Wiley \& Sons, 2011..372 p.

[18] Li J Y, Jaszczak R J, Coleman R E. A filtered backprojection algorithm for axial head motion correction in fan-beam SPECT. Physics in Medicine and Biology, 1995; 40(12): 2053-2063.

[19] Li C M, Kao C Y, Gore J C, Ding Z H. Minimization of region-scalable fitting energy for image segmentation. IEEE transactions on image processing, 2008; 17(10): 1940-1949.

[20] Gonzalez R C, Woods R E, Eddins S L. Digital Image Processing Using MATLAB, 2nd Edition. Gatesmark Publishing, 2010; 827p. 